\title{
Structural analysis and predicting effects of natural mutations on telomerase
}

\author{
Monikaben Padariya ${ }^{1}$, Umesh Kalathiya ${ }^{1}$, Maciej Baginski ${ }^{1}$ \\ ${ }^{1}$ Faculty Of Chemistry, Gdansk University Of Technology, Gdansk, Poland \\ E-mail: monikapadariya1@gmail.com
}

Telomeres, the nucleoproteins ends of eukaryotic chromosomes are essential elements which stabilize chromosome ends and allow the replication of linear DNA molecules. Specific telomere synthesis mechanisms such as telomerase enzyme solved the problem of DNA end replication, and it emerged as a new potential target for cancer treatment since this enzyme is active in about $80 \%$ of cancer cells and silent in normal somatic cells. Telomerase is a holoenzyme consisting of several subunits, including hTR (human telomerase RNA), TEP1 (telomerase associated protein 1), hTERT (human telomerase reverse transcriptase), hsp90 (heat shock protein 90), p23, and dyskerin.[1] The ability of telomerase to extend telomeric DNA length is subject to complicated controls. Not only the hTERT gene subject to transcriptional, posttranscriptional, and epigenetic control, but access of telomerase to the telomere ends is also regulated by the shelterin complex.

There are two general strategies of telomerase targeting in cancer treatment; targeting telomerase directly by inhibiting the activity of its catalytic subunit (hTERT) or its RNA template and targeting the telomerase subunit indirectly blocking telomerase access to telomeres or inhibiting binding of telomerase-associated proteins. Several synthetic compounds with telomerase inhibition (catalytic subunit) properties have been developed in recent years, the majority of these compounds are highly toxic or telomerase showed resistant towards these drugs/compounds. To understand behaviour of telomerase enzyme at molecular level, in our current work we studied natural mutants for telomerase enzyme (TERT catalytic subunit of human) and its activities towards different set of compounds using computational methods. Since crystal structure of human telomerase is not available in PDB database, we used generated structure of TERT catalytic subunit of human (using homology modeling method) based on crystal structure of the TERT catalytic subunit of Tribolium castaneum (PBD ID: 3DU6). In our previous study, considering importance of pyrazole template, a series of novel inhibitors were designed by replacing central ring of acridine with pyrazole (dibenzopyrrole).[2] Designed compounds were tested in telomerase activesite using computational methods and our in silico results are currently tested experimentally. Collecting results from our previous study [2] and data from literature, compounds which are derivatives of anthra[1,2-d]imidazole-6,11-dione, aryl$2 \mathrm{H}$-pyrazole, and dibenzopyrrole (C_9i, C_9j, and C_9k) were studied in this work. Selected compounds were docked with mutant as well as wild type human telomerase using: MOE, CDOCKER, Ligandfit docking, and AutoDock softwares. Residues Arg631 and Tyr717 when mutated in human telomerase to Gln/Pro and Gly/Phe, respectively showed distinct behavior in presence of different ligands. Mutants which reduced interaction of telomerase with ligands, were residues Pro and Phe replaced with Arg and Tyr at 631 and 717 position, respectively. Whereas, mutation of Arg631 to Gln631 and Tyr717 to Gly717 improved interaction of ligand with telomerase. Our results from docking are currently being tested using molecular dynamics simulation (Gromacs software) approach to trace dynamic behaviour of telomerase enzyme.

This work is supported by grant no 2014/13/B/NZ7/02207 from National Science Centre, Poland.

[1] Blackburn, E. H. et al. (1983) Ann. Rev. Biochem. 53, 163-194.

[2] Kalathiya, U. et al. (2014) IEEE-ACM Trans. Comput. Biol. Bioinform. 11, 1196-1207.

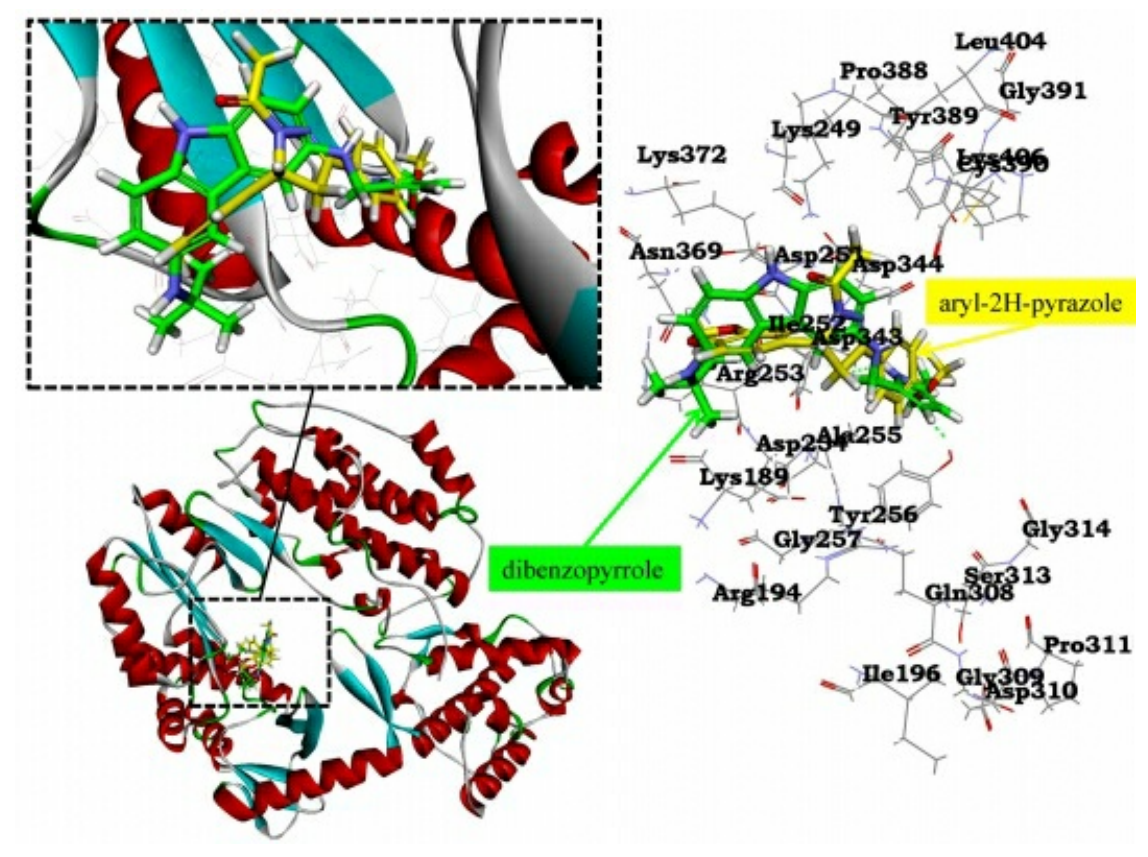

Keywords: mutation, molecular simulation, intermolecular interactions 